\title{
Electrical Characterization of Interdigitated Humidity Sensors Based on CNT Modified Calixarene Molecules
}

\author{
C. ÖZBeK ${ }^{a}$, E. Culcular $^{a}$, S. OKuR ${ }^{b, *}$, M. Yilmaz ${ }^{c}$ And M. KurT ${ }^{d}$ \\ ${ }^{a}$ Department of Physics, Faculty of Science, Izmir Institute of Technology, Gulbahce Koyu Kampusu \\ 35430, Urla, Izmir, Turkey \\ ${ }^{b}$ Material Science and Engineering, Faculty of Engineering and Architecture, Izmir Katip Celebi University \\ Cigli, İzmir, Turkey \\ ${ }^{c}$ Department of Chemistry, Selçuk University, Selçuklu/Konya, Turkey \\ ${ }^{d}$ Department of Physics, Ahi Evran University, 40100 Kirsehir, Turkey
}

\begin{abstract}
In this study, we report on the optimization and characterization of chloroform soluble calix[4]arene derivative as a humidity sensor based on electrical properties. Due to the fact that calix[4]arene molecules are nonconductive, we modified the calix[4]arene molecules with carbon nanotubes with electrostatic bond in order to increase the conductance of calix[4]arene molecule (5,11,17,23-tetra-ter-butyl-25,27-dehydrazinamidcarbonilmetoxy-26,28-dehydroxy-kalix[4]aren). Using photolithography technique, we fabricated interdigitated gold electrodes with 3 micrometers separation to investigate the electrical properties of carbon nanotubes modified calix[4]arene molecule where we used dropcast method to form a thin film of carbon nanotubes modified calix[4]arene molecule on the gold electrodes. Our reproducible experimental results indicated that the chloroform soluble carbon nanotubes modified calix[4]arene films have great potential for humidity sensing applications at room temperature operations.

DOI: 10.12693/APhysPolA.123.461

PACS: 66.30.je, 65.40.gp, 65.40.gk, 65.40.gd, 64.70.fm, 64.10. $+\mathrm{h}, 51.10 .+\mathrm{y}$
\end{abstract}

\section{Introduction}

On account of their highly tunable size- and shape-dependent chemical and physical properties, preparation of nanoscale materials for chemical and biological sensing has proven a promising avenue. The high surface area and large pore volume per unit mass, in particular, make them favorable to improve sensitivity and response times of sensors $[1,2]$. Thin films of calixarene derivatives have been widely used in chemical sensors. On account of their zeolite-like capacity and selectivity, calixarenes became promising materials for sensor applications [3-5]. Calixarenes are cyclic oligomers containing large-scaled molecular cavities, whose dimensions depend on the number of aryl fragments (usually 4, 6, 8) in macrocycle. Various functional groups can be bound to upper and lower rim. It is known that calixarenes can form "host-guest" complexes [6]. Calixarene molecule is as a "host", while metal ions and different organic compounds are as "guests". The sensitivity and selectivity of calixarene films can easily be managed by varying the number of aryl fragments and functional groups. Electrochemical sensors based on calixarenes have been shown to be sensitive to various metal ions $[7,8]$.

It is also known that especially water soluble calixarene derivatives adsorb water molecules [9]. In our previous paper, we reported on humidity sensing properties and adsorption-desorption kinetics of calixarene based on QCM techniques [3]. The working principle

* corresponding author of metal oxide or ceramic type of sensors is based on variation in electrical parameters such as resistance [10], impedance [11], and capacitance [12-14]. The signal to noise ratio of sensing device depends on the amount of physisorped i.e. absorbed molecules on the sensor element $[15,16]$.

In this work, however, electrical response of carbon nanotubes (CNT) modified calix[4]arene molecule to relative humidity $(\mathrm{RH})$ changes between various $\mathrm{RH}$ was measured to show the effect of moisture on a resistive type sensor. Our results show that CNT modified calix[4]arene molecule can be used as sensing element for calixarene based humidity sensor applications.

\section{Experimental}

CNT modified calixarene molecule was dispersed in chloroform solvent, and then $5 \mu \mathrm{L}$ of CNT modified calixarene - chloroform solution was dropped onto the interdigitated gold electrodes (IDE) electrodes using drop-cast technique. Using following steps we fabricated IDE: firstly, the glass was cleaned ultrasonically in acetone, ethanol, isopropanol and distilled water, respectively and then dried using high purity nitrogen. The cleaned substrate was first coated with $\mathrm{Cr}, 10 \mathrm{~nm}$ thick, that is used to make a strong link between gold and glass. Afterwards, $\mathrm{Cr}$ coated substrate was also coated with gold the thickness of which is $150 \mathrm{~nm}$. Using photolithography technique, we fabricated IDE with $3 \mu \mathrm{m}$ separation to investigate the electrical properties of CNT modified calix[4]arene molecule where we used dropcast method to 
form a thin film of CNT modified calix[4]arene molecule on these gold electrodes.

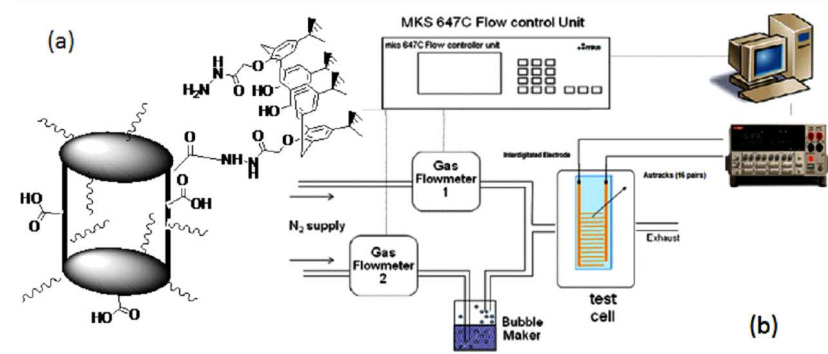

Fig. 1. Calixarene modified CNTs (a), experimental setup to measure the electrical response of CNT modified calixarene film under various relative humidity conditions between 17 and $85 \% \mathrm{RH}(\mathrm{b})$.

Figure 1 shows the experimental setup to measure the electrical response of CNT modified calixarene molecule sensing film under various relative humidity conditions between 17 and $85 \% \mathrm{RH}$. The film of CNT modified calixarene molecules between the gold electrodes were exposed to varying relative humidity conditions inside a $100 \mathrm{~cm}^{3}$ test cell by controlling the ratio of wet/dry nitrogen flow between 0 and $100 \%$ in 2 periodic steps and 50 increasing and decreasing steps to see linear response of CNT modified calixarene film to moisture using a MKS flow meter control system at flows ranging from 0 to $1000 \mathrm{sccm}$. The measurements were taken synchronously with a Keithley 2420 source meter.

\section{Results and discussions}

Figure 2a shows the electrical response of CNT modified calixarene film covered sensor during adsorption and desorption process for a period of $200 \mathrm{~s}$. An MKS flow-meter control system between 0-1000 sccm is used to control the ratio of wet/dry nitrogen flow through the test cell to vary relative humidity conditions to change concentration of moisture as shown in Fig. 1. Wet and dry nitrogen are used alternately to obtain recovery and response characteristics of the CNT modified calixarene sensing element for humidity sensing.

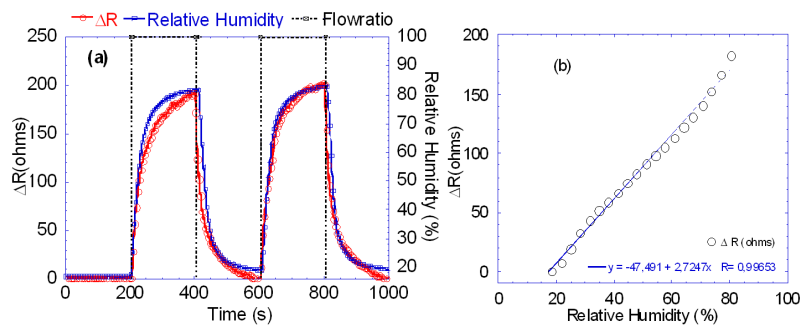

Fig. 2. The resistance changes of CNT modified calixarene molecules versus varying $\mathrm{RH}$ values.

The red and blue continuous lines represent the variation of resistance and humidity, whereas black dashed lines represent the wet/dry nitrogen flow ratio on the pilot as shown in Fig. 2. There are two types of adsorption and desorption cycles that are used to determine the electrical response of CNT modified calixarene molecule sensing element between gold finger electrodes against relative humidity changes between $17 \%$ and $85 \%$ RH. In our previous paper, we carried out similar study using both different types of calixarene and different technique called QCM [3]. In this technique, the corresponding RH values in the test cell were synchronously recorded with a Sensirion sensor as well. The Sensirion humidity sensor showed $17 \% \mathrm{RH}$, when $1000 \mathrm{sccm}$ of dry nitrogen was sent through the test cell as seen in Fig. 2a. Moreover, it showed $85 \% \mathrm{RH}$, when $1000 \mathrm{sccm}$ of wet nitrogen, acquired by passing dry nitrogen through a half filled water bubbler at constant temperature around $30^{\circ} \mathrm{C}$, was sent through the test cell. In order to see response of maximum desiccation and moistening process, only dry and wet nitrogen are sent consequently with $200 \mathrm{~s}$ periods as shown in Fig. 2a. The total resistance change of CNT modified calixarene was measured as $190.1 \Omega$, when $100 \%$ wet nitrogen with $1000 \mathrm{sccm}$ was sent at $85 \% \mathrm{RH}$. Figure 2b illustrates linear response of CNT modified calixarene electrodes for $500 \mathrm{~s}$ when the wet/dry nitrogen ratio is increased with $20 \mathrm{sccm}$ steps for an equal time intervals of $5 \mathrm{~s}$ (stepwise). The resistance of CNT modified calixarene show a clear linear response between $15 \%$ and $85 \% \mathrm{RH}$. Looking at the figures one may say that the resistance of the CNT modified calixarene thin film increases due to the increased amount of physisorped or capillary condensed water molecules as a result of increasing relative humidity.

\section{Conclusion}

A CNT modified calixarene was prepared and a resistive type humidity sensor was fabricated. The electrical response of this resistive type CNT modified sensor was measured when exposed to relative humidity between 17 and $85 \%$ at room temperature. Our results show that CNT modified calixarene molecule is a potential humidity sensing material with high periodic and nearly linear response. Furthermore, the resistance of the CNT modified calixarene has given reproducible responses to humidity changes. According to these results, it is seen that the electrical response of CNT modified calixarene molecule is quite sensitive to humidity changes and can be further investigated as humidity sensor applications.

\section{Acknowledgments}

This research was supported by TUBITAK (Turkish Scientific Association) under project number TBAG 109 T240 and IYTE research project number 2010IYTE25.

\section{References}

[1] T. Asefa, C.T. Duncan, K.K. Sharma, Analyst 134, 1980 (2009) 
[2] J. Xu, Q. Pan, Y. Shun, Z. Tianet, Sens. Actuators B, Chem. 66, 277 (2000)

[3] S. Okur, M. Kus, F. Ozel, M. Yllmaz, Sens. Actuators $B$ 145, 93 (2010)

[4] S. Korposh, R. Selyanchyn, S.-W. Lee, Sens. Actuators B, Chem. 147, 599 (2010).

[5] S. Rizzo, F. Sannicol, T. Benincori, G. Schiavon, S. Zecchin, G. Zotti, J. Mater. Chem. 14, 1804 (2004)

[6] C.D. Gutsche, Calixarenes in: Monographs in Supramolecular Chemistry, Eed. J.F. Stoddart, The Royal Society of Chemistry, Cambridge 1989, p. 172.

[7] R.J. Forster, A. Cadogan, M.T. Diaz, Sens. Actuators B 4325 (1991).

[8] R.B. Chaabane, M. Gamoudi, G. Guillaud, C. Jouve, R. Lamartine, A. Bouazizi, H. Maaref, Sens. Actuators B 31, 41 (1996)

[9] W. Yang, R. Manek, W.M. Kolling, M. Brits, W. Liebenberg, M.M.D. Villiers, Supramol. Chem. 17, 485 (2005)
[10] A. Erol, S. Okur, B. Comba, Ö. Mermer, M.C.Arikan, Sens. Actuators B Chem. 145174 (2010).

[11] R.-J. Wu, Y.L. Sun, C.C. Lin, H.W. Chen, M. Chavali, Sens. Actuators B Chem. 115, 198 (2006)

[12] R. Nahar, V. Khanna, W. Khokle, J. Phys. D, Appl. Phys. 17, 2087 (1984)

[13] U. Kang, K.D. Wise, IEEE Trans. Electron Dev. 47, 702 (2002)

[14] S. Ozturk, D. Balkose, S. Okur, J. Umemura, Colloids Surf. A-Physicochem. Eng. Aspects 302, 67 (2007)

[15] M. Kus, S. Okur, Sens. Actuators B, Chem. 143, 177 (2009)

[16] S. Okur, , M. Kus, F. Özel, V. Aybek, M. Yilmaz, Talanta 81, 248 (2010) 